\title{
Severity of low pre-pregnancy body mass index and perinatal outcomes: the Japan Environment and Children's Study
}

Kentaro Nakanishi ${ }^{1}$, Yasuaki Saijo ${ }^{2 *}$, Eiji Yoshioka ${ }^{2}$, Yukihiro Sato ${ }^{2}$, Yasuhito Kato ${ }^{1,2}$, Ken Nagaya ${ }^{3}$, Satoru Takahashi ${ }^{4}$, Yoshiya Ito ${ }^{5}$, Sumitaka Kobayashi ${ }^{6}$, Chihiro Miyashita ${ }^{6}$, Atsuko Ikeda-Araki ${ }^{6,7}$, Reiko Kishi ${ }^{6}$ and the Japan Environment and Children's Study (JECS) Group

\begin{abstract}
Background: The extremes of maternal pre-pregnancy body mass index (BMI) are known to be risk factors associated with obstetric and adverse perinatal outcomes. Among Japanese women aged 20 years or older, the prevalence of underweight (BMl $<18.5 \mathrm{~kg} / \mathrm{m}^{2}$ ) was $11.5 \%$ in 2019 . Maternal thinness is a health problem caused by the desire to become slim. This study aimed to investigate the association between the severity of maternal low pre-pregnancy BMI and adverse perinatal outcomes, including preterm birth (PTB), low birth weight (LBW), and small-for-gestational age (SGA).

Methods: We conducted a prospective cohort study using data from the Japan Environment and Children's Study, which recruited pregnant individuals between 2011 and 2014. Pre-pregnancy BMI was categorized as severe-moderate underweight $\left(\mathrm{BMI} \leq 16.9 \mathrm{~kg} / \mathrm{m}^{2}\right)$, mild underweight $\left(\mathrm{BMl}, 17.0-18.4 \mathrm{~kg} / \mathrm{m}^{2}\right)$, low-normal weight $(\mathrm{BMI}, 18.5-19.9 \mathrm{~kg} /$ $\mathrm{m}^{2}$ ), high-normal weight (BMl, $\left.20.0-22.9 \mathrm{~kg} / \mathrm{m}^{2}\right)$, overweight $\left(\mathrm{BMl}, 23.0-24.9 \mathrm{~kg} / \mathrm{m}^{2}\right)$, and obese $\left(\mathrm{BMI} \geq 25.0 \mathrm{~kg} / \mathrm{m}^{2}\right)$. The high-normal weight group was used as the reference for statistical analyses. Adjusted logistic regression was performed to evaluate the association between pre-pregnancy BMI and PTB, LBW, and SGA.

Results: Of 92,260 singleton pregnant individuals, the prevalence was $2.7 \%$ for severe-moderate underweight, $12.9 \%$ for mild underweight, and 24.5\% for low-normal weight. The prevalence of adverse outcomes was 4.6\% for PTB, 8.1\% for LBW, and 7.6\% for SGA. The adjusted odds ratios (aORs) for PTB were 1.72 (95\% confidence interval [Cl], 1.46-2.03) for severe-moderate underweight and $1.26(95 \% \mathrm{Cl}, 1.14-1.39)$ for mild underweight. The aORs of LBW were 2.55 ( $95 \% \mathrm{Cl}, 2.27-2.86)$ for severe-moderate underweight, 1.64 (95\% Cl, 1.53-1.76) for mild underweight, and $1.23(95 \% \mathrm{Cl}$, 1.16-1.31) for low-normal weight. The aORs of SGA were $2.53(95 \% \mathrm{Cl}, 2.25-2.84)$ for severe-moderate underweight, $1.66(95 \% \mathrm{Cl}, 1.55-1.79)$ for mild underweight, and 1.29 (95\% Cl, 1.21-1.38) for low-normal weight.
\end{abstract}

Conclusions: A dose-response relationship was found between the severity of low pre-pregnancy BMI and PTB, LBW, and SGA. Even low-normal BMI $\left(18.5-19.9 \mathrm{~kg} / \mathrm{m}^{2}\right)$ increased the risk of LBW and SGA. This study provides useful information for pre-conception counseling in lean individuals.

*Correspondence: y-saijo@asahikawa-med.ac.jp

${ }^{2}$ Division of Public Health and Epidemiology, Department of Social

Medicine, Asahikawa Medical University, 1-1-1, Midorigaoka higashi2-jo,

Asahikawa, Hokkaido 078-8510, Japan

Full list of author information is available at the end of the article

(c) The Author(s) 2022. Open Access This article is licensed under a Creative Commons Attribution 4.0 International License, which permits use, sharing, adaptation, distribution and reproduction in any medium or format, as long as you give appropriate credit to the original author(s) and the source, provide a link to the Creative Commons licence, and indicate if changes were made. The images or other third party material in this article are included in the article's Creative Commons licence, unless indicated otherwise in a credit line to the material. If material is not included in the article's Creative Commons licence and your intended use is not permitted by statutory regulation or exceeds the permitted use, you will need to obtain permission directly from the copyright holder. To view a copy of this licence, visit http://creativecommons.org/licenses/by/4.0/. The Creative Commons Public Domain Dedication waiver (http://creativeco mmons.org/publicdomain/zero/1.0/) applies to the data made available in this article, unless otherwise stated in a credit line to the data. 
Keywords: Adverse perinatal outcomes, Low birth weight, Maternal body mass index, Preterm birth, Small-forgestational age

\section{Background}

The extremes of maternal pre-pregnancy body mass index (BMI) are known to be risk factors associated with obstetric and adverse perinatal outcomes [1-7]. Higher maternal pre-pregnancy BMI increases the risk of hypertensive disorders during pregnancy, gestational diabetes, preterm birth (PTB), fetal death, stillbirth, large-for-gestational age, and macrosomia [2-7]. A lower maternal pre-pregnancy BMI increases the risk of $\mathrm{PTB}$, low birth weight (LBW), and small-for-gestational age (SGA) [1, 3-11].

In Japan, maternal underweight is a common health problem because many individuals in the reproductiveage strongly desire to be slim and work towards it [12]. Among Japanese women aged 20 years or older, the prevalence of underweight individuals $\left(<18.5 \mathrm{~kg} / \mathrm{m}^{2}\right)$ was 11.5\% in the 2019 National Health and Nutrition Survey of Japan [13]. In particular, this figure among Japanese women in their 20 's was $20.7 \%$. Indeed, the frequency of LBW has been increasing in Japan despite the rate of PTB being as low as 5.6\% in 2019 [14]. Several studies reported that low maternal pre-pregnancy BMI was significantly associated with adverse perinatal outcomes, such as PTB, LBW, and SGA [1, 4, 5, 7-11, 15]. However, to our knowledge, no studies on the severity of low prepregnancy BMI have been conducted in East Asian countries including Japan where pregnancy-aged individuals have a lower BMI compared to other regions. Further, no prospective cohort studies examining the adverse obstetric and perinatal outcomes associated with severity of pre-pregnancy underweight were found. Although two large cohort studies in Japan reported the association between pre-pregnancy underweight and PTB and SGA, the severity of maternal pre-pregnancy underweight was not evaluated $[3,16]$.

The definition of BMI in Asia differs from that in the United States and European countries because the prevalence of underweight pregnant individuals in Asia is higher than that in other countries [17]. The appropriate BMI categories recommended by the World Health Organization (WHO) for Asian populations are as follows: less than $18.5 \mathrm{~kg} / \mathrm{m}^{2}$, underweight; $18.5-23 \mathrm{~kg} / \mathrm{m}^{2}$, increasing but acceptable risk; $23-27.5 \mathrm{~kg} / \mathrm{m}^{2}$, increased risk; and $\geq 27.5 \mathrm{~kg} / \mathrm{m}^{2}$, high risk [17]. The WHO also states that countries should use all BMI categories, with a view to facilitate international comparisons as follows: the trigger points are 18.5, 20, 23, 25, 27.5, 30, and $32.5 \mathrm{~kg} / \mathrm{m}^{2}$ [17]. Thus, BMI needs to be classified according to this WHO categorization to conduct the study on pre-pregnancy BMI in Asian populations.

Additionally, few studies have quantified the association between maternal pre-pregnancy BMI and adverse perinatal outcomes, using intuitive methods such as the restricted cubic spline (RCS). The RCS has the advantages of being able to characterize a dose-response association between a continuous exposure and an outcome, and check the assumption of linearity of the association visually and statistically [18].

The aim of the present study was to investigate the association between the severity of maternal low prepregnancy BMI and gestational age at delivery and neonatal birth weight using a nationwide, prospective cohort study of approximately 100,000 mothers and neonates. In addition, we used the RCS to analyze the association between maternal pre-pregnancy BMI and adverse perinatal outcomes, including PTB, LBW, and SGA.

\section{Methods}

\section{Study design and participants}

We conducted a prospective cohort study using the dataset jecs-ta-20190930, which was released in October 2019, from the Japan Environment and Children's Study (JECS). The JECS is an ongoing nationwide birth cohort study, the details of which have already been published $[19,20]$. The JECS mainly aimed to investigate the association between environmental factors and children's health and development by recruiting pregnant individuals from 15 Regional Centers between January 2011 and March 2014. However, in 2013, when recruitment was largely stabilized, approximately only $45 \%$ of children could be assessed [20].

Between January 2011 and March 2014, 104,062 fetal recodes were enrolled in the JECS. After excluding miscarriage $(n=1254)$, stillbirth $(n=382)$, and unknown birth outcome $(n=2122)$, the present study included 100,304 live births. Then, after excluding pregnancies in the same participants, the study involved 94,754 participants. Finally, after excluding multiple pregnancies $(n=1809)$, and pregnancies with chromosomal abnormality $(n=207)$, missing value of maternal prepregnancy BMI $(n=126)$, missing value of gestational age at delivery $(n=283)$, and missing value of neonatal birth weight $(n=69)$, the number of study participants was 92,260 singleton, pregnant individuals (Fig. 1). The number of participants in the SGA analyses decreased to 92,041 because we excluded 219 deliveries that were 


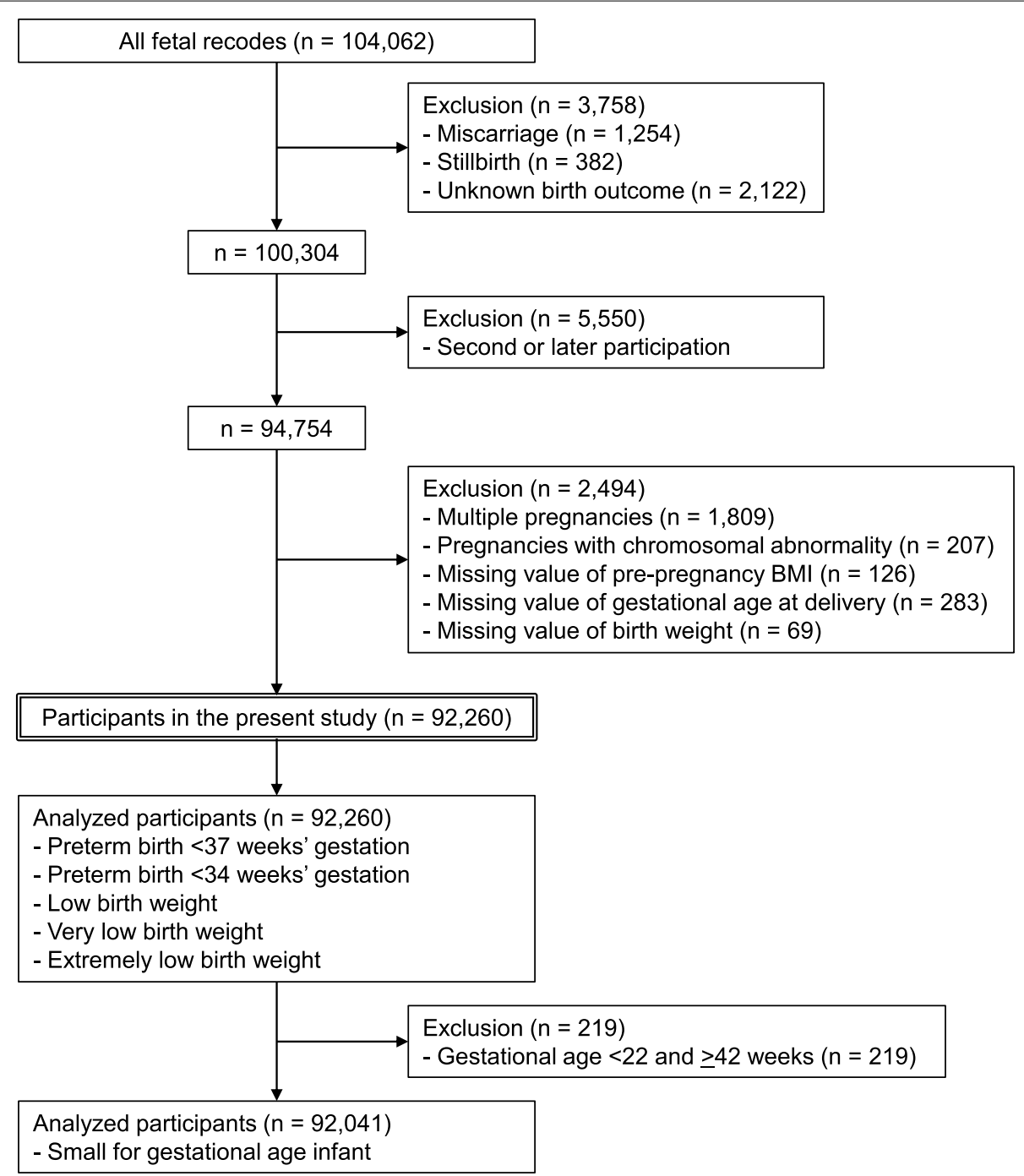

Fig. 1 Flow diagram of the study participants

performed at a gestational age of $<22$ weeks or $>41$ weeks (Fig. 1).

\section{Ethics}

The study protocol was reviewed and approved by the Institutional Review Board on Epidemiological Studies of the Ministry of the Environment and by the Ethics Committees of all participating institutions (No. 100910001) [19]. This study was conducted in accordance with the Declaration of Helsinki. All participants in the JECS provided written informed consent.

\section{Maternal and neonatal baseline information}

Maternal baseline information, including maternal educational background, smoking status, and alcohol consumption was obtained from self-administered questionnaires provided to the enrolled pregnant individuals during the second/third trimester. The following information was also collected from their medical record transcripts: parity, assisted reproductive technology, multiple births, maternal history of preterm birth less than 37 weeks of gestation, medical histories of hypertension, diabetes mellitus, autoimmune disease, thyroid disease such as hyperthyroidism and hypothyroidism, neonate's date of birth, and gestational period. Although maternal height and pre-pregnancy weight were, in principle, obtained from medical record transcripts, in instances where the above information (maternal height and pre-pregnancy weight) was missing in medical record transcripts, the values were obtained from self-reports of participants. The 
mother's age at the time of childbirth was calculated from the mother's and the neonate's birth dates.

Pre-pregnancy BMI was defined as the maternal prepregnancy weight divided by the square of maternal height obtained from medical record transcripts or selfreports. Pregnant individuals were categorized based on their pre-pregnancy BMI as follows: severe-moderate underweight $\left(\mathrm{BMI} \leq 16.9 \mathrm{~kg} / \mathrm{m}^{2}\right)$, mild underweight (BMI, 17.0-18.4 kg/m²), low-normal weight (BMI, 18.5$19.9 \mathrm{~kg} / \mathrm{m}^{2}$ ), high-normal weight (BMI, 20.0-22.9 kg/ $\mathrm{m}^{2}$ ), overweight (BMI, $23.0-24.9 \mathrm{~kg} / \mathrm{m}^{2}$ ), and obese $\left(B M I \geq 25.0 \mathrm{~kg} / \mathrm{m}^{2}\right)$, with reference to the WHO's classification for BMI $[12,21]$. The high-normal pre-pregnancy BMI group was the reference group for statistical analyses.

Parity was categorized as $0,1,2$, or higher. Maternal smoking status was defined as follows: never or previously did, but quit before recognizing current pregnancy; previously did, but quit after identifying current pregnancy, and; yes, I still smoke. Maternal alcohol consumption was defined as follows: never or previously did, but quit before identifying current pregnancy; previously did, but quit after identifying current pregnancy, and; yes, I drink. The highest level of education of the mother was defined as follows: junior high school, high school, technical junior college, technical/vocational college or associate degree, bachelor's degree, or Graduate degree (Master's/Doctor'). Medical information of the mother and neonate, including maternal age at delivery, gestational age at delivery, neonatal birth weight, and neonatal sex, was transcribed from the medical record transcripts at delivery.

\section{Outcomes}

The main outcomes of the present study were the incidence of adverse perinatal outcomes, including PTB, very preterm birth (VPTB), LBW, very low birth weight (VLBW), extremely low birth weight (ELBW), and SGA. PTB and VPTB were defined as the gestational age of less than 37 weeks and 34 weeks at delivery, respectively. LBW, VLBW, and ELBW were defined as neonatal birth weights of less than $2500 \mathrm{~g}, 1500 \mathrm{~g}$, and $1000 \mathrm{~g}$, respectively. SGA was defined as a birth weight below the 10th percentile, accounting for infant sex, parity, and gestational age according to the Japan Pediatric Society [22], and the percentiles were calculated using Excel-based clinical tools for growth evaluation of children distributed by the Japanese Society for Pediatric Endocrinology [23].

\section{Statistical analysis}

The adjusted odds ratio (OR) and 95\% confidence interval (CI) for adverse perinatal outcomes were calculated by maternal pre-pregnancy BMI compared to the reference group (BMI, $20.0-22.9 \mathrm{~kg} / \mathrm{m}^{2}$, high-normal weight) using a multivariable logistic regression model, adjusted for maternal age at delivery, parity (except for SGA analysis), assisted reproductive technology, maternal smoking status, maternal alcohol consumption, maternal educational background, maternal history of preterm birth, and the medical history of hypertension, diabetes mellitus, autoimmune disease, and thyroid disease such as hyperthyroidism and hypothyroidism.

The RCSs were used to detect a dose-response relationship between pre-pregnancy BMI and each adjusted OR of the adverse perinatal outcomes. The RCS models the association between predictor and outcome using cubic polynomials and linear terms [24]. The RCS requires the placement of a number of knots along the predictor value range. Usually, three to five knots are sufficient to allow for complex associations between predictors and outcomes; the present study was analyzed with five knots placed at the 5th, 27.5th, 50th, 72.5th, and 95th percentiles of the BMI distribution.

Because the dataset had some missing values, based on the assumption of missing at random, the k-nearest neighbor ( $\mathrm{kNN}$ ) imputation method of the $\mathrm{R}$ package "VIM" (ver. 4.1.2; R Foundation for Statistical Computing) was used [25], introducing all outcomes and adjusted variables, along with gestational weeks. The $\mathrm{kNN}$ imputation method is a widely accepted single imputation method, and its validity has been confirmed [26]. The kNN imputation was appropriate for the RCS analysis. In addition, we performed a sensitivity analysis using the complete dataset and excluded all missing values. The results of the sensitivity analysis are shown in the Supplemental Table. Statistical significance was set at a twotailed $P$ value of $<0.05$. All analyses, except k-nearest neighbor imputation, were performed using STATA version 16.1, for Windows (Stata Corporation, College Station, TX, USA).

\section{Results}

In the present study, $99.8 \%(92,089 / 92,260)$ of the maternal pre-pregnancy weights were obtained from medical record transcripts and $0.2 \%(171 / 92,260)$ from self-reported data. Of the participants who had both sets of data, $65.4 \%$ reported the same weight in both sets. The high-normal weight group (BMI, $20.0-22.9 \mathrm{~kg} / \mathrm{m}^{2}$ ), which was the reference group, had the highest prevalence $(38.5 \%)$ among all pre-pregnancy BMI categories (Table 1). The prevalence of low maternal pre-pregnancy BMI was as follows: $15.7 \%(14,448 / 92,260)$ for underweight $\left(\mathrm{BMI} \leq 18.4 \mathrm{~kg} / \mathrm{m}^{2}\right)$ and $24.5 \%(22,583 / 92,260)$ for low-normal weight (BMI, $18.5-19.9 \mathrm{~kg} / \mathrm{m}^{2}$ ). Of pre-pregnant individuals who were underweight, the prevalence 
Table 1 Maternal and neonatal characteristics $(n=92,260)$

\begin{tabular}{|c|c|}
\hline Characteristics & Values $^{\mathrm{a}}$ \\
\hline \multicolumn{2}{|l|}{ Maternal age (years) } \\
\hline$\leq 24$ & $9252(10.0)$ \\
\hline $25-29$ & $25,374(27.5)$ \\
\hline $30-34$ & $32,543(35.3)$ \\
\hline $35-39$ & $20,838(22.6)$ \\
\hline$\geq 40$ & $4247(4.6)$ \\
\hline Missing & $6(0.01)$ \\
\hline \multicolumn{2}{|l|}{ Parity } \\
\hline 0 & $38,291(41.5)$ \\
\hline 1 & $33,694(36.5)$ \\
\hline$\geq 2$ & $17,985(19.5)$ \\
\hline Missing & $2290(2.5)$ \\
\hline \multicolumn{2}{|l|}{ Assisted reproductive technology } \\
\hline No & $89,068(96.5)$ \\
\hline Yes & $2761(3.0)$ \\
\hline Missing & $431(0.5)$ \\
\hline \multicolumn{2}{|l|}{ Pre-pregnancy BMI } \\
\hline$\leq 16.9$, Severe to moderate underweight & $2515(2.7)$ \\
\hline 17.0-18.4, Mild underweight & $11,933(12.9)$ \\
\hline 18.5-19.9, Low-normal weight & $22,583(24.5)$ \\
\hline 20.0-22.9, High-normal weight & $35,537(38.5)$ \\
\hline 23.0-24.9, Overweight & $9658(10.5)$ \\
\hline$\geq 25.0$, Obese & $10,034(10.9)$ \\
\hline \multicolumn{2}{|l|}{ Smoking during pregnancy } \\
\hline No & $73,263(79.4)$ \\
\hline Quit after pregnancy & $12,636(13.7)$ \\
\hline Yes & $4103(4.4)$ \\
\hline Missing & $2258(2.5)$ \\
\hline \multicolumn{2}{|l|}{ Drinking during pregnancy } \\
\hline No & $44,991(48.8)$ \\
\hline Quit after pregnancy & $42,529(46.1)$ \\
\hline Yes & $2497(2.7)$ \\
\hline Missing & $2243(2.4)$ \\
\hline \multicolumn{2}{|l|}{ Maternal educational background } \\
\hline Junior high school & $4326(4.7)$ \\
\hline High school & $28,318(30.7)$ \\
\hline $\begin{array}{l}\text { Technical junior college or technical/vocational } \\
\text { college }\end{array}$ & $37,991(41.2)$ \\
\hline University or above & $19,601(21.2)$ \\
\hline Missing & $2024(2.2)$ \\
\hline \multicolumn{2}{|l|}{ History of preterm birth } \\
\hline No & $86,753(94.0)$ \\
\hline Yes & $2853(3.1)$ \\
\hline Missing & $2654(2.9)$ \\
\hline \multicolumn{2}{|l|}{ Hypertension } \\
\hline No & $89,408(96.9)$ \\
\hline Yes & $1162(1.3)$ \\
\hline Missing & $1690(1.8)$ \\
\hline \multicolumn{2}{|l|}{ Diabetes mellitus } \\
\hline No & $89,571(97.1)$ \\
\hline
\end{tabular}

Table 1 (continued)

\begin{tabular}{ll}
\hline Characteristics & Values $^{\mathbf{a}}$ \\
\hline Yes & $995(1.1)$ \\
Missing & $1694(1.8)$ \\
Autoimmune disease & \\
No & $90,377(98.0)$ \\
Yes & $183(0.2)$ \\
Missing & $1700(1.8)$ \\
Thyroid disease & \\
No & $89,344(96.8)$ \\
Yes & $1224(1.3)$ \\
Missing & $1692(1.8)$ \\
Gestational age at delivery (weeks) & $39(22-43)$ \\
Birth weight (grams) & $3028(312-5214)$ \\
Neonatal sex & \\
Male & $47,329(51.3)$ \\
Female & $44,924(48.7)$ \\
Missing & $7(0.01)$ \\
Preterm birth (<37 weeks' gestation) & $4284(4.6)$ \\
Very preterm birth $(<34$ weeks' gestation) & $907(1.0)$ \\
Low birth weight $(<2500 \mathrm{~g})$ & $7514(8.1)$ \\
Very low birth weight $(<1500 \mathrm{~g})$ & $528(0.6)$ \\
Extremely low birth weight $(<1000 \mathrm{~g})$ & $228(0.3)$ \\
Small-for-gestational age & \\
No & $85,029(92.2)$ \\
Yes & $7012(7.6)$ \\
Missing & $219(0.2)$ \\
\hline avles are &
\end{tabular}

${ }^{a}$ Values are presented as $\mathrm{n}(\%)$ or median (range)

was $17.4 \%(2515 / 14,448)$ for severe-moderate underweight $\left(\mathrm{BMI} \leq 16.9 \mathrm{~kg} / \mathrm{m}^{2}\right)$ and $82.6 \%(11,933 / 14,448)$ for mild underweight (BMI, $17.0-18.4 \mathrm{~kg} / \mathrm{m}^{2}$ ).

In the total study population, the prevalence of PTB was $4.6 \%$ and that of VPTB was $1.0 \%$ (Table 1). Regarding neonatal birth weight, the prevalence of LBW was $8.1 \%$, of VLBW was $0.6 \%$, of ELBW was $0.3 \%$, and of SGA was 7.6\% (Table 1).

Low maternal pre-pregnancy BMI was significantly associated with PTB, LBW, and SGA (Table 2). Regarding low pre-pregnancy BMI, the aORs of $\mathrm{PTB}$ were 1.72 (95\% CI, 1.46-2.03; $P<0.001$ ) for severe-moderate underweight and 1.26 (95\% CI, 1.14-1.39; $P<0.001)$ for mild underweight. The aORs of LBW were 2.55 (95\% CI, 2.27-2.86; $P<0.001$ ) for severe-moderate underweight, 1.64 (95\% CI, 1.53-1.76; $P<0.001)$ for mild underweight, and 1.23 (95\% CI, 1.16-1.31, $P<0.001)$ for low-normal weight. The aORs of SGA were 2.53 (95\% CI, 2.25-2.84; $P<0.001$ ) for severemoderate underweight, 1.66 (95\% CI, 1.55-1.79; $P<0.001)$ for mild underweight, and 1.29 (95\% CI, 1.21-1.38; $P<0.001$ ) for low-normal weight (Table 2 ). 
Table 2 Odds ratios of maternal pre-pregnancy body mass index for adverse perinatal outcomes after imputation $(n=92,260)$

\begin{tabular}{|c|c|c|c|c|c|c|}
\hline \multirow[t]{2}{*}{ Outcomes } & \multicolumn{6}{|c|}{ Pre-pregnancy body mass index $\left(\mathrm{kg} / \mathrm{m}^{2}\right)$} \\
\hline & $\leq 16.9$ & $17.0-18.4$ & $18.5-19.9$ & $20.0-22.9$ & $23.0-24.9$ & $25.0 \leq$ \\
\hline \multicolumn{7}{|l|}{ PTB } \\
\hline$N(\%)$ & $171(3.99)$ & $604(14.10)$ & $960(22.41)$ & $1477(34.48)$ & $449(10.48)$ & $623(14.54)$ \\
\hline Crude OR (95\% Cl) & $1.68(1.43-1.98)$ & $1.23(1.12-1.35)$ & $1.02(0.94-1.11)$ & reference & $1.12(1.01-1.25)$ & $1.53(1.39-1.68)$ \\
\hline Adjusted OR $(95 \% \mathrm{Cl})^{\text {a }}$ & $1.72(1.46-2.03)$ & $1.26(1.14-1.39)$ & $1.05(0.96-1.14)$ & reference & $1.07(0.96-1.20)$ & $1.36(1.23-1.51)$ \\
\hline \multicolumn{7}{|l|}{ VPTB } \\
\hline$N(\%)$ & $29(3.20)$ & $112(12.35)$ & $181(19.96)$ & $304(33.52)$ & $112(12.35)$ & $169(18.63)$ \\
\hline Crude OR (95\% Cl) & $1.35(0.92-1.98)$ & $1.10(0.88-1.37)$ & $0.94(0.78-1.13)$ & reference & $1.36(1.09-1.69)$ & $1.99(1.64-2.40)$ \\
\hline Adjusted OR $(95 \% \mathrm{Cl})^{\text {a }}$ & $1.36(0.92-2.00)$ & $1.12(0.90-1.39)$ & $0.96(0.79-1.15)$ & reference & $1.28(1.03-1.59)$ & $1.72(1.42-2.09)$ \\
\hline \multicolumn{7}{|l|}{ LBW } \\
\hline N (\%) & $404(5.38)$ & $1297(17.26)$ & $1896(25.23)$ & $2499(33.26)$ & $678(9.02)$ & $740(9.85)$ \\
\hline Crude OR (95\% Cl) & $2.53(2.26-2.84)$ & $1.61(1.50-1.73)$ & $1.21(1.14-1.29)$ & reference & $1.00(0.91-1.09)$ & $1.05(0.97-1.15)$ \\
\hline Adjusted OR $(95 \% \mathrm{Cl})^{\text {a }}$ & $2.55(2.27-2.86)$ & $1.64(1.53-1.76)$ & $1.23(1.16-1.31)$ & reference & $0.96(0.88-1.05)$ & $0.96(0.88-1.04)$ \\
\hline \multicolumn{7}{|l|}{ VLBW } \\
\hline N (\%) & $17(3.22)$ & $69(13.07)$ & $104(19.70)$ & $168(31.82)$ & $65(12.31)$ & 105 (19.89) \\
\hline Crude OR (95\% Cl) & $1.43(0.87-2.36)$ & $1.22(0.92-1.62)$ & $0.97(0.76-1.24)$ & reference & $1.43(1.07-1.90)$ & $2.23(1.74-2.84)$ \\
\hline Adjusted OR $(95 \% \mathrm{Cl})^{\text {a }}$ & $1.45(0.88-2.40)$ & $1.25(0.5-1.66)$ & $1.00(0.78-1.27)$ & reference & $1.33(1.00-1.78)$ & $1.82(1.41-2.35)$ \\
\hline \multicolumn{7}{|l|}{ ELBW } \\
\hline$N(\%)$ & $5(2.19)$ & $28(12.28)$ & $45(19.74)$ & $83(36.40)$ & $23(10.09)$ & $44(19.30)$ \\
\hline Crude OR (95\% Cl) & $0.85(0.34-2.10)$ & $1.00(0.65-1.54)$ & $0.85(0.59-1.23)$ & reference & $1.02(0.64-1.62)$ & $1.88(1.30-2.71)$ \\
\hline Adjusted OR $(95 \% \mathrm{Cl})^{\text {a }}$ & $0.84(0.34-2.07)$ & $1.03(0.67-1.58)$ & $0.87(0.61-1.25)$ & reference & $0.95(0.60-1.51)$ & $1.56(1.07-2.27)$ \\
\hline \multicolumn{7}{|l|}{$S G A^{b}$} \\
\hline N (\%) & $381(5.43)$ & $1245(17.76)$ & $1871(26.68)$ & $2349(33.50)$ & $605(8.63)$ & $561(8.00)$ \\
\hline Crude OR (95\% Cl) & $2.52(2.24-2.83)$ & $1.64(1.53-1.77)$ & $1.27(1.20-1.36)$ & reference & $0.94(0.86-1.04)$ & $0.84(0.76-0.92)$ \\
\hline Adjusted OR $(95 \% \mathrm{Cl})^{\text {a }}$ & $2.53(2.25-2.84)$ & $1.66(1.55-1.79)$ & $1.29(1.21-1.38)$ & reference & $0.92(0.84-1.01)$ & $0.78(0.71-0.86)$ \\
\hline
\end{tabular}

The adjusted odds ratios with statistical significance are presented in bold

${ }^{a}$ The odds ratio compared to that of infants of mothers with the high-normal pre-pregnancy body mass index $\left(20.0-22.9 \mathrm{~kg} / \mathrm{m}^{2}\right)$, adjusted for maternal age at delivery, parity (except for SGA analysis), assisted reproductive technology, maternal smoking status, maternal alcohol consumption, maternal educational background, history of preterm birth, medical history of hypertension, diabetes mellitus, autoimmune disease, and thyroid disease

${ }^{b}$ The total number of participants was 92,041

In the complete dataset, maternal low pre-pregnancy BMI was also significantly associated with PTB, LBW, and SGA (Supplemental Table 1).

For VPTB, VLBW, and ELBW, significant differences were identified in maternal pre-pregnancy obese group compared with the reference group (Table 2). However, no significant difference was found between the low pre-pregnancy BMI groups.

Figure 2 demonstrates the non-linear association between maternal pre-pregnancy BMI and adverse perinatal outcomes using 5 knots RCS with adjustment for confounders. The dose-response relationship between pre-pregnancy BMI and PTB, LBW, and SGA was observed only in the low BMI range (Fig. 2A, $\mathrm{C}, \mathrm{F})$. The RCS also showed a U-shaped relationship between maternal pre-pregnancy BMI and VPTB and VLBW (Fig. 2B, D).

\section{Discussion}

We further divided low pre-pregnancy BMI into two levels $\left(<16.9 \mathrm{~kg} / \mathrm{m}^{2}\right.$, and $\left.17.0-18.4 \mathrm{~kg} / \mathrm{m}^{2}\right)$ and analyzed the association between the two levels of low BMI and low-normal BMI $\left(18.5-19.9 \mathrm{~kg} / \mathrm{m}^{2}\right)$ and adverse perinatal outcomes. To our knowledge, this is the first study to evaluate the association between the severity of maternal low pre-pregnancy BMI and adverse perinatal outcomes in Japanese women, in whom a high prevalence of thinness can be observed. The dose-response relationship between the severity of low pre-pregnancy BMI and PTB, LBW, and SGA was found only in the low BMI range. In addition, the present study focused on adverse perinatal outcomes, such as earlier PTB and LBW. However, the association between low maternal pre-pregnancy BMI and VPTB, VLBW, and ELBW was not statistically significant. 

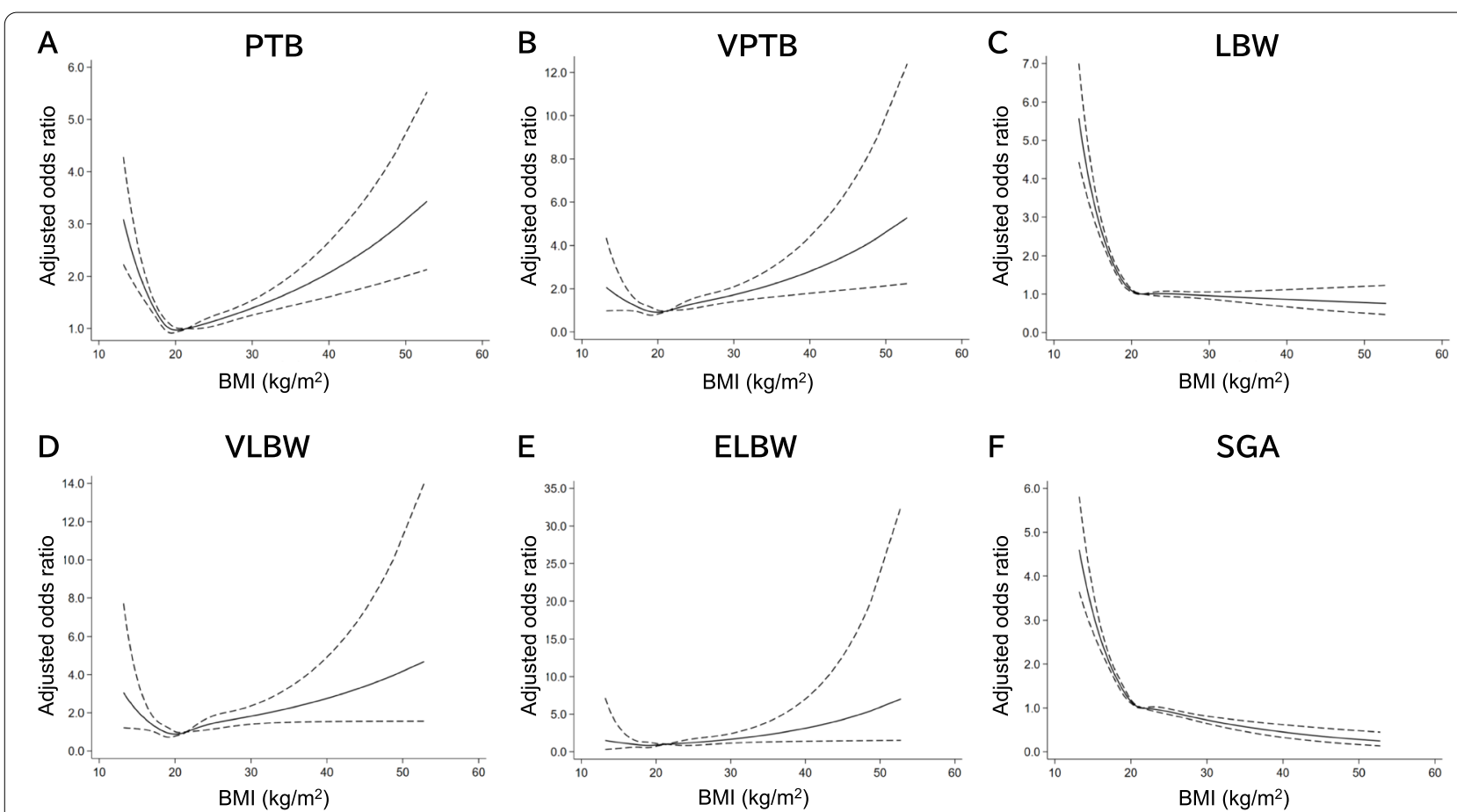

Fig. 2 Maternal pre-pregnancy BMI and the risk of adverse perinatal outcomes using restricted cubic spline. Values are adjusted odds ratio and 95\% confidence interval compared with the referent group (high-normal weight, 20.0-22.4 kg/ $\mathrm{m}^{2}$ ), adjusted for maternal age at delivery, parity (except for small-for-gestational age [SGA] analysis), assisted reproductive technology, maternal smoking status, maternal alcohol consumption, maternal educational background, history of preterm birth, and the medical history of hypertension, diabetes mellitus, autoimmune disease, and thyroid disease. The adjusted odds ratios are shown as solid lines. The $95 \%$ confidence interval is indicated by dotted lines. A PTB: preterm delivery before 37 weeks of gestation. B VPTB: preterm delivery before 34 weeks of gestation. C LBW: low birth weight $\mathbf{D}$ VLBW: very low birth weight. E ELBW: extremely low birth weight. F SGA: small-for-gestational age. BMI, body mass index

The association between underweight pre-pregnant individuals and PTB has been well studied previously $[1,5,9-11,15]$. However, only a few studies have been reported on the association between the severity of low pre-pregnancy BMI and PTB [1,9-11]. There are four studies that demonstrated that the severe and moderate pre-pregnant underweight groups significantly increased the risk of PTB compared with normal weighing groups [1,9-11]. Except for the study by Lynch et al. [1], the mildly underweight pre-pregnant group was associated with increased risk of PTB in three studies [9-11]. Moreover, a meta-analysis on the association between pre-pregnancy BMI and pregnancy complications also demonstrated that underweight prepregnant individuals were at higher risk of experiencing PTB [5]. In the present study, the pre-pregnancy underweight group was at a significantly higher risk of PTB compared with the high-normal weight group. Regarding the severity of low pre-pregnancy BMI, our results show that the lower the pre-pregnancy BMI, the greater the risk of PTB. Therefore, our results are consistent with those of previous studies.
In the present study, underweight pre-pregnant individuals were not significantly associated with an increased risk of PTB before 34 weeks of gestation compared with the high-normal weight group. In the study by Shaw et al. [15], there was no significant association between maternal pre-pregnancy underweight and spontaneous PTB before 32 weeks of gestation. However, Salihu et al. [9] and Ratnasiri et al. [6] reported that maternal pre-pregnancy underweight was significantly associated with an increased risk of PTB before 33 and 32 weeks of gestation, respectively. Interestingly, Ratnasiri et al. [6] reported that the aOR was lower for PTB before 32 weeks of gestation than for PTB before 37 weeks of gestation. This finding, stating that low pre-pregnancy BMI had a smaller impact on early PTB, was similar to our results. In the present study, earlier PTB was strongly associated with higher pre-pregnancy BMI than lower pre-pregnancy BMI. However, we found that the risk of VPTB tended to increase with lower pre-pregnancy BMI because the RCS showed a $\mathrm{U}$-shaped relationship between low pre-pregnancy BMI and VPTB. 
The association between maternal pre-pregnancy underweight and neonatal birth weight has been reported in many studies $[3,5,6,8,27-31]$; however, only one study by Salmon et al. [11] investigated the association between the severity of low pre-pregnancy BMI and neonatal birth weight. They reported that severe-moderate and mild underweight maternal pre-pregnancy BMIs were significantly associated with an increased risk of intrauterine growth restriction, LBW, and VLBW with a dose-response relationship. They reported that the value of aOR was lower for birth weights less than $1500 \mathrm{~g}$ than for those weighing less than $2500 \mathrm{~g}$, which is similar to the results of our study. In addition, the meta-analysis by Santos et al. [5] demonstrated that a lower pre-pregnancy BMI was associated with an increased risk of SGA. The present study demonstrated that the risk of LBW and SGA was significantly associated with low pre-pregnancy BMI, including low-normal BMI $(18.5-19.9 \mathrm{~kg} /$ $\mathrm{m}^{2}$ ). The dose-response relationship between the severity of maternal low pre-pregnancy BMI and LBW as well as SGA was represented in the low BMI range by RCS analysis. Although VLBW and ELBW were not statistically associated with maternal low pre-pregnancy BMI, a $\mathrm{U}$-shaped relationship between pre-pregnancy BMI and VPTB and VLBW was observed in the RCS.

Our findings confirmed that the lower the pre-pregnancy BMI, the greater the risk of PTB, LBW, and SGA among Asian populations. Moreover, even low-normal pre-pregnancy BMI increased the risk of LBW and SGA. Therefore, in countries with a high prevalence of lean individuals, pre-pregnancy weight control in lean individuals of reproductive age can be an important intervention to prevent PTB, LBW, and SGA.

However, VPTB, VLBW, and ELBW were strongly associated with a higher pre-pregnancy BMI than lower BMI in our study. The present study analyzed these results based on maternal variables at the first prenatal visit and did not consider adverse complications during pregnancy. Therefore, VPTB, VLBW, and ELBW may be affected by adverse pregnancy complications because high pre-pregnancy BMI increases the risk of hypertensive disorders of pregnancy and gestational diabetes mellitus.

As in previous studies, the present study confirmed that maternal low pre-pregnancy BMI was an independent factor associated with PTB because a significant difference was identified after adjusting for confounding factors associated with adverse perinatal outcomes. Several studies have reported that maternal undernutrition, maternal infection, and inflammation due to lack of nutrients necessary for the immune system contribute to the relationship between low pre-pregnancy BMI and PTB [1, 9, 28]. However, it remains unclear why maternal low pre-pregnancy BMI increases the risk of PTB. Further research is needed to reveal the mechanism by which low pre-pregnancy BMI affects PTB.

The present study had several strengths. First, this was a large prospective cohort study that included pregnant Japanese individuals, with a high prevalence of thinness. Most previous studies on low pre-pregnancy BMI have been conducted in the United States and European countries, where the prevalence of thinness is low. Second, maternal pre-pregnancy BMI was categorized according to the appropriate BMI categories in the Asian population. In addition, the normal weight group was divided into two groups: low-normal weight and high-normal weight. Third, the RCS analysis was used to represent the non-linear relationship between maternal low pre-pregnancy BMI and adverse perinatal outcomes.

However, this study had several limitations. First, maternal gestational weight gain (GWG) was not included in the models because it was on the pathway from pre-pregnancy BMI to the outcomes. Future studies should note that the results of this study may not be representative of the general population due to the recent changes in the GWG guidelines. A meta-analysis on GWG [32] has reported that the optimal weight gain range was $14.0 \mathrm{~kg}$ to less than $16.0 \mathrm{~kg}$ for underweight individuals. However, this study has also reported that maternal pre-pregnancy BMI was more strongly associated with adverse maternal and infant outcomes than the amount of GWG. Also, dietary intake during pregnancy was not included in the models. Dietary intake during pregnancy, as well as GWG, may be related with PTB, LBW, and SGA. Second, any maternal complications that may have occurred during pregnancy were not considered as confounders because this study was conducted to predict the risk of adverse perinatal outcomes a pregnant individuals had at the time of her first visit. Third, a part of the maternal pre-pregnancy BMI was calculated using self-reported data when maternal height and pre-pregnancy weight were not available in medical record transcripts. Forth, the numbers of VPTB, VLBW, and ELBW were relatively small. Since our results demonstrated a trend toward increased risk of VPTB and VLBW with lower pre-pregnancy BMI, an increase in the number of cases may show a significant difference in adverse outcomes. Further research is needed to reveal the association between maternal low pre-pregnancy BMI and VPTB, VLBW, and ELBW. Fifth, this study may not apply to the non-Asian population as it was conducted on Japanese women. 


\section{Conclusion}

The present study demonstrated the association between the severity of maternal low pre-pregnancy BMI and adverse perinatal outcomes, including PTB, LBW, and SGA, in Japanese pregnant individuals. The dose-response relationship between the severity of low pre-pregnancy BMI and adverse perinatal outcomes was found in the low BMI range. Even low-normal prepregnancy BMI increased the risk of LBW and SGA. To reduce the risk of PTB, LBW, and SGA in lean individuals, it would be better to maintain a high-normal prepregnancy BMI. This study provides useful information for pre-conception counseling in lean individuals.

\begin{abstract}
Abbreviations
BMI: Body mass index; Cl: Confidence interval; ELBW: Extremely low birth weight; GWG: Gestational weight gain; JECS: Japan Environment and Children's Study; kNN: k-nearest neighbor; LBW: Low birth weight; OR: Odds ratio; PTB: Preterm birth; RCS: Restricted cubic spline; SGA: Small-for-gestational age; VLBW: Very low birth weight; VPTB: Very preterm birth; WHO: World Health Organization.
\end{abstract}

\section{Supplementary Information}

The online version contains supplementary material available at https://doi. org/10.1186/s12884-022-04418-3.

Additional file 1: Supplemental Table 1. Odds ratios of maternal prepregnancy body mass index in the complete dataset $(n=84,366)$.

\section{Acknowledgements}

We would like to express our gratitude to all JECS study participants and all staff members involved in the data collection. Members of the JECS Group as of 2021: Michihiro Kamijima (principal investigator, Nagoya City University, Nagoya, Japan), Shin Yamazaki (National Institute for Environmental Studies, Tsukuba, Japan), Yukihiro Ohya (National Center for Child Health and Development, Tokyo, Japan), Nobuo Yaegashi (Tohoku University, Sendai, Japan), Koichi Hashimoto (Fukushima Medical University, Fukushima, Japan), Chisato Mori (Chiba University, Chiba, Japan), Shuichi Ito (Yokohama City University, Yokohama, Japan), Zentaro Yamagata (University of Yamanashi, Chuo, Japan), Hidekuni Inadera (University of Toyama, Toyama, Japan), Takeo Nakayama (Kyoto University, Kyoto, Japan), Hiroyasu Iso (Osaka University, Suita, Japan), Masayuki Shima (Hyogo College of Medicine, Nishinomiya, Japan), Youichi Kurozawa (Tottori University, Yonago, Japan), Narufumi Suganuma (Kochi University, Nankoku, Japan), Koichi Kusuhara (University of Occupational and Environmental Health, Kitakyushu, Japan), and Takahiko Katoh (Kumamoto University, Kumamoto, Japan).

Consortia the Japan Environment and Children's Study (JECS) Group. Michihiro Kamijima ${ }^{8}$, Shin Yamazaki ${ }^{9}$, Yukihiro Ohya ${ }^{10}$, Nobuo Yaegashi ${ }^{11}$, Koichi Hashimoto ${ }^{12}$, Chisato Mori ${ }^{13}$, Shuichi Ito ${ }^{14}$, Zentaro Yamagata ${ }^{15}$, Hidekuni Inadera ${ }^{16}$, Takeo Nakayama ${ }^{17}$, Hiroyasu Iso ${ }^{18}$, Masayuki Shima ${ }^{19}$, Youichi Kurozawa ${ }^{20}$, Narufumi Suganuma ${ }^{21}$, Koichi Kusuhara ${ }^{22}$, and Takahiko Katoh $^{23}$.

${ }^{8}$ Nagoya City University, Nagoya, Japan. ${ }^{9}$ National Institute for Environmental Studies, Tsukuba, Japan. ${ }^{10}$ National Center for Child Health and Development, Tokyo, Japan. ${ }^{11}$ Tohoku University, Sendai, Japan. ${ }^{12}$ Fukushima Medical University, Fukushima, Japan. ${ }^{13}$ Chiba University, Chiba, Japan. ${ }^{14}$ Yokohama City University, Yokohama, Japan. ${ }^{15}$ University of Yamanashi, Chuo, Japan. ${ }^{16}$ University of Toyama, Toyama, Japan. ${ }^{17}$ Kyoto University, Kyoto, Japan. ${ }^{18}$ Osaka University, Suita, Japan. ${ }^{19}$ Hyogo College of Medicine, Nishinomiya, Japan. ${ }^{20}$ Tottori University, Yonago, Japan. ${ }^{21}$ Kochi University, Nankoku, Japan. ${ }^{22}$ University of Occupational and Environmental Health, Kitakyushu, Japan. ${ }^{23}$ Kumamoto University, Kumamoto, Japan.

\section{Authors' contributions}

KN and YS designed the research. YS, EY, KN, ST, YI, SK, CM, Al and RK collected data. $K N$ and $Y S$ conducted data analysis. $K N$ and $Y S$ contributed to data interpretation. YS, EY, YS, YK, KN, ST, YI, SK, CM, Al and RK conducted critical reviews. KN drafted the initial manuscript. YS made critical revisions. All authors reviewed and commented upon the manuscript. All authors approved the final manuscript.

\section{Funding}

This study was funded by the Ministry of the Environment, Japan. The findings and conclusions of this article are solely the responsibility of the authors and do not represent the official views of the above government.

\section{Availability of data and materials}

Data are unsuitable for public deposition due to ethical restrictions and legal framework of Japan. It is prohibited by the Act on the Protection of Personal Information (Act No. 57 of 30 May 2003, amendment on 9 September 2015) to publicly deposit the data containing personal information. Ethical Guidelines for Medical and Health Research Involving Human Subjects enforced by the Japan Ministry of Education, Culture, Sports, Science and Technology and the Ministry of Health, Labour and Welfare also restricts the open sharing of the epidemiologic data. All inquiries about access to data should be sent to: jecs-en@nies.go.jp. The person responsible for handling enquiries sent to this e-mail address is Dr. Shoji F. Nakayama, JECS Programme Office, National Institute for Environmental Studies.

\section{Declarations}

Ethics approval and consent to participate

The study protocol was reviewed and approved by the Institutional Review Board on Epidemiological Studies of the Ministry of the Environment and by the Ethics Committees of all participating institutions (No. 100910001). This study was conducted in accordance with the Declaration of Helsinki. All participants in the JECS provided written informed consent.

\section{Consent for publication}

Not applicable.

\section{Competing interests}

The authors declare that they have no competing interests.

\section{Author details}

${ }^{1}$ Department of Obstetrics and Gynecology, Asahikawa Medical University, 1-1-1, Midorigaoka higashi2-jo, Asahikawa, Hokkaido 078-8510, Japan. ${ }^{2}$ Division of Public Health and Epidemiology, Department of Social Medicine, Asahikawa Medical University, 1-1-1, Midorigaoka higashi2-jo, Asahikawa, Hokkaido 078-8510, Japan. ${ }^{3}$ Division of Neonatology, Perinatal Medical Center, Asahikawa Medical University Hospital, 1-1-1, Midorigaoka higashi2-jo, Asahikawa, Hokkaido 078-8510, Japan. ${ }^{4}$ Department of Pediatrics, Asahikawa Medical University, 1-1-1, Midorigaoka higashi2-jo, Asahikawa, Hokkaido 078-8510, Japan. ${ }^{5}$ Faculty of Nursing, Japanese Red Cross Hokkaido College of Nursing, 664-1, Akebono-cho, Kitami, Hokkaido 090-0011, Japan. ${ }^{6}$ Center for Environmental and Health Sciences, Hokkaido University, Kita12-jo, Nishi7-chome, Kita-ku, Sapporo, Hokkaido 060-0812, Japan. ${ }^{7}$ Faculty of Health Sciences, Hokkaido University, Kita12-jo, Nishi5-chome, Kita-ku, Sapporo, Hokkaido 060-0812, Japan.

Received: 30 October 2021 Accepted: 21 January 2022

Published online: 11 February 2022

\section{References}

1. Lynch AM, Hart JE, Agwu OC, Fisher BM, West NA, Gibbs RS. Association of extremes of prepregnancy BMI with the clinical presentations of preterm birth. Am J Obstet Gynecol. 2014;210(5):428.e421-9. https://doi.org/10. 1016/j.ajog.2013.12.011.

2. Aune D, Saugstad OD, Henriksen T, Tonstad S. Maternal body mass index and the risk of fetal death, stillbirth, and infant death: a systematic review 
and meta-analysis. JAMA. 2014;311(15):1536-46. https://doi.org/10.1001/ jama.2014.2269.

3. Enomoto K, Aoki S, Toma R, Fujiwara K, Sakamaki K, Hirahara F. Pregnancy outcomes based on pre-pregnancy body mass index in Japanese women. PLoS One. 2016;11(6):e0157081. https://doi.org/10.1371/journal. pone. 0157081.

4. Liu P, Xu L, Wang Y, Zhang Y, Du Y, Sun Y, et al. Association between perinatal outcomes and maternal pre-pregnancy body mass index. Obes Rev. 2016;17(11):1091-102. https://doi.org/10.1111/obr.12455.

5. Santos $\mathrm{S}$, Voerman E, Amiano P, Barros H, Beilin $\sqcup$, Bergström A, et al. Impact of maternal body mass index and gestational weight gain on pregnancy complications: an individual participant data metaanalysis of European, North American and Australian cohorts. BJOG. 2019;126(8):984-95. https://doi.org/10.1111/1471-0528.15661.

6. Ratnasiri AWG, Lee HC, Lakshminrusimha S, Parry SS, Arief VN, DeLacy $\mathrm{IH}$, et al. Trends in maternal prepregnancy body mass index (BMI) and its association with birth and maternal outcomes in California, 2007-2016: a retrospective cohort study. Plos One. 2019;14(9):e0222458. https://doi. org/10.1371/journal.pone.0222458.

7. Tang J, Zhu X, Chen Y, Huang D, Tiemeier $H$, Chen $\mathrm{R}$, et al. Association of maternal pre-pregnancy low or increased body mass index with adverse pregnancy outcomes. Sci Rep. 2021;1 1(1):3831. https://doi.org/10.1038/ s41598-021-82064-z.

8. Ronnenberg AG, Wang $X$, Xing H, Chen C, Chen D, Guang W, et al. Low preconception body mass index is associated with birth outcome in a prospective cohort of Chinese women. J Nutr. 2003;133(11):3449-55. https://doi.org/10.1093/jn/133.11.3449.

9. Salihu HM, Mbah AK, Alio AP, Clayton HB, Lynch O. Low pre-pregnancy body mass index and risk of medically indicated versus spontaneous preterm singleton birth. Eur J Obstet Gynecol Reprod Biol. 2009;144(2):11923. https://doi.org/10.1016/j.ejogrb.2009.02.047.

10. Girsen Al, Mayo JA, Carmichael SL, Phibbs CS, Shachar BZ, Stevenson DK, et al. Women's prepregnancy underweight as a risk factor for preterm birth: a retrospective study. BJOG. 2016;123(12):2001-7. https://doi.org/ 10.1111/1471-0528.14027.

11. Salmon C, Thibon P, Prime L, Renouf S, Dreyfus M, Dolley P. Impact of maternal underweight on obstetric and neonatal prognosis: a retrospective study. Eur J Obstet Gynecol Reprod Biol. 2020;260:6-9. https://doi. org/10.1016/j.jogrb.2020.12.040.

12. Suzuki S. Current prevalence of and obstetric outcomes in underweight Japanese women. PLoS One. 2019;14(6):e0218573. https://doi.org/10. 1371/journal.pone.0218573.

13. Japanese Ministry of Health, Labour and Welfare. National Health and Nutrition survey in Japan. 2019; Available at: https://www.e-stat.go.jp/ stat-search/files?page $=1$ \&layout $=$ datalist\&touke $=00450171 \&$ tstat $=$ $000001041744 \&$ cycle $=7 \&$ year $=20190 \&$ month $=0 \&$ tclass $1=0000011485$ 07. Accessed 24 April 2021 (in Japanese).

14. Japanese Ministry of Health, Labour and Welfare. Vital Statistics of Japan 2019; Available at: https://www.e-stat.go.jp/stat-search/files?page $=1 \&$ layout $=$ datalist\&touke $=00450011$ \&tstat $=000001028897 \&$ cycle $=7 \&$ year $=20190 \&$ month $=0 \&$ tclass $1=000001053058 \&$ tclass $2=0000010530$ $61 \& t c l a s s 3=000001053064 \&$ result_back $=1 \&$ tclass 4 val $=0$. Accessed 24 April 2021 (in Japanese).

15. Shaw GM, Wise PH, Mayo J, Carmichael SL, Ley C, Lyell DJ, et al. Maternal prepregnancy body mass index and risk of spontaneous preterm birth. Paediatr Perinat Epidemiol. 2014;28(4):302-11. https://doi.org/10.1111/ ppe.12125.

16. Morisaki N, Nagata C, Jwa SC, Sago H, Saito S, Oken E, et al. Pre-pregnancy BMI-specific optimal gestational weight gain for women in Japan. J Epidemiol. 2017;27(10):492-8. https://doi.org/10.1016/j.je.2016.09.013.

17. Appropriate body-mass index for Asian populations and its implications for policy and intervention strategies. Lancet. 2004;363(9403):157-63. https://doi.org/10.1016/S0140-6736(03)15268-3.

18. Desquilbet $L$, Mariotti F. Dose-response analyses using restricted cubic spline functions in public health research. Stat Med. 2010;29(9):1037-57. https://doi.org/10.1002/sim.3841.

19. Kawamoto T, Nitta H, Murata K, Toda E, Tsukamoto N, Hasegawa M, et al. Rationale and study design of the Japan environment and children's study (JECS). BMC Public Health. 2014;14:25. https://doi.org/10.1186/ 1471-2458-14-25
20. Michikawa T, Nitta H, Nakayama SF, Yamazaki S, Isobe T, Tamura K, et al. Baseline profile of participants in the Japan environment and Children's study (JECS). J Epidemiol. 2018;28(2):99-104. https://doi.org/10.2188/jea. JE20170018.

21. Physical status: the use and interpretation of anthropometry. Report of a WHO expert committee. World Health Organ Tech Rep Ser. 1995;854:1-452.

22. Itabashi K, Fujimura M, Kusuda S. Introduction of the new standard for birth size by gestational ages. J Jpn Pediatr Soc. 2010;114:1271-93 (in Japanese).

23. The Japanese Society for Pediatric Endocrinology. Excel-based Clinical Tools for Growth Evaluation of Children; Available at: http://jspe.umin.jp/ medical/chart_dl.html. Accessed 30 March 2021 (in Japanese).

24. Nieboer D, Vergouwe Y, Roobol MJ, Ankerst DP, Kattan MW, Vickers AJ, et al. Nonlinear modeling was applied thoughtfully for risk prediction: the prostate biopsy collaborative group. J Clin Epidemiol. 2015;68(4):426-34. https://doi.org/10.1016/j.jclinepi.2014.11.022.

25. Kowarik A, Templ M. Imputation with the R package VIM. J Stat Softw. 2016;74(7):1-16. https://doi.org/10.18637/jss.v074.i07.

26. Jerez JM, Molina I, García-Laencina PJ, Alba E, Ribelles N, Martín M, et al. Missing data imputation using statistical and machine learning methods in a real breast cancer problem. Artif Intell Med. 2010;50(2):105-15. https://doi.org/10.1016/j.artmed.2010.05.002.

27. Sebire NJ, Jolly M, Harris J, Regan L, Robinson S. Is maternal underweight really a risk factor for adverse pregnancy outcome? A population-based study in London. BJOG. 2001;108(1):61-6. https://doi.org/10.1111/j.14710528.2001.00021.x.

28. Fujiwara K, Aoki S, Kurasawa K, Okuda M, Takahashi T, Hirahara F. Associations of maternal pre-pregnancy underweight with small-for-gestationalage and spontaneous preterm birth, and optimal gestational weight gain in Japanese women. J Obstet Gynaecol Res. 2014;40(4):988-94. https:// doi.org/10.1111/jog.12283.

29. Murai U, Nomura K, Kido M, Takeuchi T, Sugimoto M, Rahman M. Prepregnancy body mass index as a predictor of low birth weight infants in Japan. Asia Pac J Clin Nutr. 2017;26(3):434-7. https://doi.org/10.6133/ apjcn.032016.11.

30. Zhao RF, Zhou L, Zhang WY. Identifying appropriate pre-pregnancy body mass index classification to improve pregnancy outcomes in women of childbearing age in Beijing, China: a retrospective cohort study. Asia Pac J Clin Nutr. 2019;28(3):567-76. https://doi.org/10.6133/apjcn.201909_28(3). 0016.

31. Tanaka T, Ashihara K, Nakamura M, Kanda T, Fujita D, Yamashita Y, et al. Associations between the pre-pregnancy body mass index and gestational weight gain with pregnancy outcomes in Japanese women. J Obstet Gynaecol Res. 2014;40(5):1296-303. https://doi.org/10.1111/jog. 12353.

32. Voerman E, Santos S, Inskip H, Amiano P, Barros H, Charles MA, et al. Association of Gestational Weight Gain with Adverse Maternal and Infant Outcomes. JAMA. 2019;321(17):1702-15. https://doi.org/10.1001/jama. 2019.3820.

\section{Publisher's Note}

Springer Nature remains neutral with regard to jurisdictional claims in published maps and institutional affiliations. 